\section{Role of Wheat Varieties and Insecticide Applications against Aphids for Better Wheat Crop Harvest}

\author{
Mahreen Yahya, Noor Abid Saeed*, Sajid Nadeem, Muhammad Hamed \\ and Sajid Shokat \\ Nuclear Institute for Agriculture and Biology, Jhang Road, Faisalabad, Pakistan
}
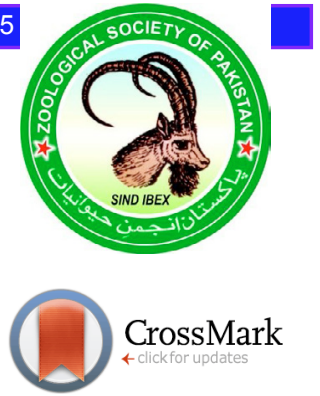

\begin{abstract}
A B S T R A C T
Wheat (Triticum aestivum L.), is a major cereal and staple food crop which plays the vital role in the economy of Pakistan. This wheat crop suffers a heavy toll in yield due to aphid attack, which affects plant vitality and grain production. Planting resistant/tolerant varieties against pests is the main way to overcome these field losses. Present study is an effort to screen different wheat varieties/lines against aphids under field conditions. Wheat varieties, Galaxy-13, Millat-11, Lasani-08, Faisalabad-08, and two wheat lines NW-1-8183-8 and NW-3-3341-7 were sown at Nuclear Institute for Agriculture and Biology (NIAB), Faisalabad during the crop season 2015-2016. The aphid infestation started in mid-January on all wheat varieties at stem elongation stage and increased gradually with the growth of plants. Three aphid species were recorded i.e., Rhopalosiphum padi (L.), Schizaphis graminum (R.) and Sitobion avenae (F.). Mean seasonal aphid population (no. of aphids/tiller) on wheat plants during the whole season was the highest in NW-1-8183-8 and NW-3-3341-7 and the lowest in Faisalabad-08. However, grain yield was the highest in Galaxy-13 and the lowest was in Lasani-08 variety. Two applications of insecticide, (imidacloprid@625 ml/hectare),significantly controlled the aphid population and enhanced the yield of all wheat varieties. The higher aphid infestations in untreated plots resulted in less chlorophyll content and reduced photosynthetic activity. Photosynthetic rate and chlorophyll contents were significantly higher in insecticide treated plots. Maximum cost-benefit ratio was found (1:9.2) in NW-1-8183-8 when treated against aphids. Wheat varieties, Galaxy-13 and Faisalabad-08 were found tolerant against aphid damage. Wheat variety Lasani- 08 should be avoided because it was found susceptible to aphid infestations, and give low net yield. It is inferred that infestation of aphids significantly reduced the grain yield when not protected by insecticide. Insecticide protected crops give maximum net returns. Integration of varietal resistance and insecticides showed an effective reduction of aphids and gave a significant increase in grain yield.
\end{abstract}

\begin{tabular}{l} 
Article Information \\
Received 25 November 2016 \\
Revised 12 March 2017 \\
Accepted 21 July 2017 \\
Available online 24 November 2017 \\
Authors' Contribution \\
\hline MY did the experiments and data \\
observations. NAS conceived, designed \\
and drafted the field study. SS \\
analyzed the data statistically. SN and \\
MH critically edited the manuscript. \\
Key words \\
Wheat varieties, Aphid, Insecticide, \\
Grain yield, Cost and benefit ratio.
\end{tabular}

\section{INTRODUCTION}

$\mathrm{G}$ lobally, the utilization of wheat (Triticum aestivum L.) is reported as $65 \%$ of grain in human food, $21 \%$ as animal feed, $8 \%$ as seed and $6 \%$ for industrial processing (Khan et al., 2009). Pakistan is the $8^{\text {th }}$ largest wheat producer worldwide (FAOSTAT, 2015), by cultivating an area of 9.180 million hectares (Anonymous, 2015). Yield per hectare in Pakistan is far less than many wheat producing countries. Yield losses, due to feeding of insect pests particularly the aphids, are massive in wheat crop (Akhtar et al., 2010; Khan et al., 2011; John et al., 2017).

Aphids (Homoptera: Aphididae) are the major economic insect-pests that cause yield losses in different crops worldwide, especially in temperate regions

\footnotetext{
* Corresponding author: noor.niab@gmail.com 0030-9923/2017/0006-2217 \$ 9.00/0

Copyright 2017 Zoological Society of Pakistan
}

(Blackman and Eastop, 2000). Damage by aphids to cereal crops are much alarming particularly in wheat crop, though stay for a short period of time, but they have fast multiplication rate and have potential to demolish the crop within few days (Jarosik et al., 2003). Aphids are known as the highest damaging pest on wheat crop in Pakistan (Khan et al., 2011). The abundance of aphid on plants results in less chlorophyll level, decreased plant transpiration (Ahmed and Nasir, 2001) and reduced photosynthetic activity (Burd and Elliott, 1996). Infested plants suffer with leave curling, delayed head emergence and improper grains with reduced grain weight (Ciepiela, 1993). Sitobion avenae (English grain aphid), Rhopalosiphum padi (Bird cherry-oat aphid), and Schizaphis graminum (Green-bug) are reported on wheat in Pakistan (Shahid et al., 2012; John et al., 2017). Occasionally, infestations of $R$. maidis were also reported on wheat (Khan, 2005).

Due to aphid feeding, variable yield losses are reported in wheat crop. Yield losses can be up to 7.9 to 
$34.2 \%$ with aphid infestation (Akhtar et al., 2010). Direct sucking of aphid can cause $10-50 \%$ losses while, indirect losses can be $20-80 \%$ (Trdan and Milevoj, 1999). For instance, the yield loss can be 35 to $40 \%$ with the presence of 15 aphids tiller ${ }^{-1}$ (Brewer and Elliott, 2004) whereas, 34 to $67 \%$ decrease in harvest is possible if aphid born viral diseases spread in wheat crop (Riedell et al., 2003).

Plant growth stages directly influence the growth, development and fecundity of insect-pest (Yazdani and Agarwal, 1997). In case of aphids, infestations occur in less numbers in earlier plant growth stages and gradually increase during vegetative growth stage of crop, peaked at heading stage and gradually decline with crop maturity (Aheer et al., 2006; Khan et al., 2012). Aphid infestation between ear emergence and flowering can cause maximum loss in yield that may reach up to $14 \%$ (Liu et al., 1986). Therefore, the early detection and management of aphid incidence is important.

Host plant resistance is an important part in managing aphids (Khattak et al., 2007; Khan et al., 2011) which can aid to overcome the economic losses (Akhtar et al., 2006). Utilization of resistant wheat varieties along with judicial use of appropriate insecticides is a better option to manage aphid infestations in wheat crop (Iqbal, 2003; Wains et al., 2010).

The objectives of the present study were: to screen wheat varieties/lines against aphid infestations under sprayed and unsprayed conditions; to determine wheat yield losses; and to estimate cost and benefit ratio of insecticides used against aphids to save crop yield harvests.

\section{MATERIALS AND METHODS}

\section{Experiment and crop husbandry}

During 2015-16, the experiment was conducted in the research area, Nuclear Institute for Agriculture and Biology (NIAB) Faisalabad. Four commercial varieties of wheat viz., Galaxy-13, Millat-11, Lasani-08, Faisalabad-08, and two wheat lines, NW-1-8183-8, and NW-3-3341-7 developed at NIAB, were sown on 28 November, 2015. The experiment was laid out in split plot design with three replicates. Wheat varieties were kept in main plots and insecticide sprays in sub-plots. The subplot area was $4.9 \times 1.8 \mathrm{~m}^{2}$. Sowing was done by hand drill, using recommended seed rate $(125 \mathrm{~kg}$ per hectare). Seeds of each entry were planted in strips of adjacent plots with 6 rows distanced at $30.5 \mathrm{~cm}$. Inter plot distance was $122 \mathrm{~cm}$. Standard agronomic practices were done uniformly throughout the experiment. Diammonium phosphate (DAP: $50 \mathrm{~kg}$ bag having $46 \% \mathrm{P}$ and $18 \% \mathrm{~N}$ ) and Urea (50 $\mathrm{kg}$ having $46 \% \mathrm{~N}$ ) were used at recommended rate of 2.5 bags per hectare. In addition to pre-sowing irrigation, three irrigations were given to crop. Weeds were managed by using three herbicides during the season. Firstly, Stomp ${ }^{\circledR}$ 330EC (Pendimethalin by FMC United Pakistan) @ 2.5 liter/hectare was done as a pre-sowing herbicides on November 28, 2015. Second herbicide, Buctril Super ${ }^{\mathbb{R}}$ 600 EC (Bromoxynil + MCPA by Bayer Pakistan) @ $750 \mathrm{ml} /$ hectare, was applied on December 29th 2015 as post emergence against broadleaf weeds. Third herbicide, Axial $^{\circledR}$ 100EC (Pinoxanden and safener cloquintocetmexyl by Syngenta Pakistan) @ $825 \mathrm{ml} /$ hectare, was applied on January 26, 2016 as post emergence against narrow leaf weeds.

\section{Insecticidal treatments and data observations \\ Two insecticidal sprays, Confidor $^{\circledR}$ 20SL} (Imidacloprid by Bayer Pakistan) @ $625 \mathrm{ml} /$ hectare, were applied to manage aphids at fourteen days interval. Applications were done on $9^{\text {th }}$ Feb. and $23^{\text {rd }}$ Feb., 2016 by using knapsack hand sprayer with water volume as 250 liters/hectare. Data collection was initiated at appearance of aphids till maturity of crop. Aphids were scouted from randomly selected 6 tillers per plot at 7 days interval. During data collection, the growth stage (GS) of the crop was also recorded, as illustrated by Laycock (2004), in order to observe onset and progress of aphid infestations with crop phenology. At each observation, aphid samples were brought to the laboratory for species wise identification. Available taxonomic keys were used to identify collected aphid species according to Blackman and Eastop (2000).

For the determination of photosynthetic rate $(A)$, a battery operated instrument Steady-State Porometer (Li1600; LI-COR Inc., Lincoln, NE) was used. Stomatal conductance and transpiration rate were measured as described by Ashraf et al. (1992). Flag leaves were kept under the sensor of instrument until the stable readings were given on the screen. Chlorophyll content was measured by a hand-held device, atLEAF+ chlorophyll meter by using flag leaves. The chlorophyll meter gives the relative amount of chlorophyll content by measuring the absorbance in two wavelength regions (660 and $940 \mathrm{~nm}$ ). The LCD displayed the measurement number and value. Photosynthetic rate and chlorophyll contents were assessed on at GS 55 (50\% emergence of ears).

Grain yield (g) samples were taken by harvesting of 10 tillers (from 0.6 sq. $\mathrm{ft}$ area) from randomly selected 3 spots (30 tillers) per plot. These tillers from each replicate were threshed and weighed separately. The difference of grain yield data $(\mathrm{g})$ from treated and untreated plots were recorded separately and yield data were converted to $\mathrm{kg}$ per hectare. 
Table I.- Occurrence of aphid infestation (aphids/tiller) in relation to wheat crop phenology.

\begin{tabular}{|c|c|c|c|c|c|c|}
\hline \multirow[t]{2}{*}{ Varieties } & \multicolumn{2}{|c|}{ Incidence on 19 Jan } & \multicolumn{2}{|c|}{ Peak infestation at different dates } & \multicolumn{2}{|c|}{ Decline on 9 Mar } \\
\hline & Crop phenology & Infestation* & Crop phenology & $\begin{array}{l}\text { Infestation } \\
\end{array}$ & Crop phenology & Infestation \\
\hline Galaxy-13 & $\begin{array}{l}\text { Start of stem } \\
\text { elongation (GS30) }\end{array}$ & 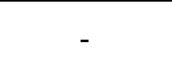 & $\begin{array}{l}\text { Booting stage } \\
\text { (GS 49) on } 2^{\text {nd }} \text { March }\end{array}$ & $26.0 \pm 0.4$ & $\begin{array}{l}\text { Ear 50\% emerged } \\
\text { (GS 55) }\end{array}$ & $4.2 \pm 0.2$ \\
\hline Millat-11 & $\begin{array}{l}\text { Start of stem } \\
\text { elongation (GS30) }\end{array}$ & - & $\begin{array}{l}\text { Booting stage } \\
\text { (GS 49) on } 2^{\text {nd }} \text { March }\end{array}$ & $25.7 \pm 0.5$ & $\begin{array}{l}\text { Ear } 50 \% \text { emerged } \\
\text { (GS 55) }\end{array}$ & $1.7 \pm 0.5$ \\
\hline NW-1-8183-8 & $\begin{array}{l}\text { Start of stem } \\
\text { elongation (GS30) }\end{array}$ & - & $\begin{array}{l}\text { Booting stage } \\
\text { (GS 49) on } 2^{\text {nd }} \text { March }\end{array}$ & $18.5 \pm 0.5$ & $\begin{array}{l}\text { Ear } 50 \% \text { emerged } \\
\text { (GS 55) }\end{array}$ & $0.3 \pm 0.2$ \\
\hline NW-3-3341-7 & $\begin{array}{l}\text { Start of stem } \\
\text { elongation (GS30) }\end{array}$ & - & $\begin{array}{l}\text { Flag leaf fully emerged } \\
\left(\text { GS39) on } 23^{\text {rd }} \mathrm{Feb}\right.\end{array}$ & $19.2 \pm 0.3$ & $\begin{array}{l}\text { Ear 50\% emerged } \\
\text { (GS 55) }\end{array}$ & $0.2 \pm 0.1$ \\
\hline Lasani-08 & $\begin{array}{l}\text { Start of stem } \\
\text { elongation (GS30) }\end{array}$ & - & $\begin{array}{l}\text { Flag leaf just visible } \\
\text { (GS37) on } 16^{\text {th }} \text { February }\end{array}$ & $21.0 \pm 0.1$ & $\begin{array}{l}\text { Ear 50\% emerged } \\
\text { (GS 55) }\end{array}$ & $0.9 \pm 0.1$ \\
\hline Faisalabad -08 & $\begin{array}{l}\text { Start of stem } \\
\text { elongation (GS30) }\end{array}$ & - & $\begin{array}{l}\text { Flag leaf fully emerged } \\
\text { (GS39) on } 23 \text { February }\end{array}$ & $11.2 \pm 0.3$ & $\begin{array}{l}\text { Ear } 50 \% \text { emerged } \\
\text { (GS 55) }\end{array}$ & $1.1 \pm 0.5$ \\
\hline
\end{tabular}

*negligibly small; GS, growth stages of wheat plant; S, Seeding; 11, first leaf; 13, Three leaves; 21, Start of tillering stage; 25, five tillers; 29, End of tillering stage; 30, Start of stem elongation; 31, First node visible; 32, Second node visible; 37, Flag leaf just visible; 39, Flag leaf fully emerged; 49, Booting; 55, Ear 50\% emerged; 59, Ears fully emerged; 69, End of flowering; 89, Fully ripe.

Table II.- Mean number of different aphid species on wheat crop during crop year 2015-16.

\begin{tabular}{|c|c|c|c|c|c|c|c|c|}
\hline $\begin{array}{l}\text { Aphid } \\
\text { Species (S) }\end{array}$ & $\begin{array}{l}\text { Varieties } \\
\text { (V) }\end{array}$ & $\begin{array}{l}\text { 2/2/16 } \\
\text { GS } 32 \\
\end{array}$ & $\begin{array}{l}\text { 9/2/16 } \\
\text { GS } 32 \\
\end{array}$ & $\begin{array}{c}\text { 16/2/16 } \\
\text { GS } 37 \\
\end{array}$ & $\begin{array}{c}23 / 2 / 16 \\
\text { GS } 39 \\
\end{array}$ & $\begin{array}{l}\text { 2/3/16 } \\
\text { GS } 49 \\
\end{array}$ & $\begin{array}{l}\text { 9/3/16 } \\
\text { GS } 55 \\
\end{array}$ & $\begin{array}{c}\text { Seasonal } \\
\text { Mean }\end{array}$ \\
\hline \multirow[t]{7}{*}{ R. padi } & V1 & $0.1 \pm 0.0$ & $0.6 \pm 0.2 \mathrm{~b}$ & $3.1 \pm 0.1 \mathrm{~h}$ & $0.8 \pm 0.0 \mathrm{f}$ & $0.8 \pm 0.0 \mathrm{e}$ & - & $1.1 \pm 0.1 \mathrm{j}$ \\
\hline & $\mathrm{V} 2$ & $0.3 \pm 0.2$ & $1.4 \pm 0.1 \mathrm{a}$ & $3.6 \pm 0.0 \mathrm{~g}$ & $0.7 \pm 0.1 \mathrm{f}$ & $0.8 \pm 0.0 \mathrm{e}$ & - & $1.4 \pm 0.1 \mathrm{ij}$ \\
\hline & V3 & $0.3 \pm 0.2$ & $1.0 \pm 0.1 \mathrm{a}$ & $3.2 \pm 0.0 \mathrm{gh}$ & $2.7 \pm 0.0 \mathrm{e}$ & $0.6 \pm 0.0 \mathrm{e}$ & - & $1.6 \pm 0.0 \mathrm{ij}$ \\
\hline & V4 & $0.1 \pm 0.0$ & $1.2 \pm 0.0 \mathrm{a}$ & $3.5 \pm 0.0 \mathrm{gh}$ & $3.8 \pm 0.1 \mathrm{~d}$ & $0.5 \pm 0.0 \mathrm{e}$ & - & $1.8 \pm 0.0 \mathrm{i}$ \\
\hline & V5 & $0.0 \pm 0.0$ & $0.5 \pm 0.0 \mathrm{~b}$ & $4.2 \pm 0.0 \mathrm{f}$ & $2.3 \pm 0.1 \mathrm{e}$ & $0.3 \pm 0.0 \mathrm{e}$ & - & $1.5 \pm 0.0 \mathrm{ij}$ \\
\hline & V6 & $0.3 \pm 0.1$ & $0.7 \pm 0.4 \mathrm{a}$ & $1.8 \pm 0.0 \mathrm{i}$ & $2.8 \pm 0.1 \mathrm{de}$ & $0.3 \pm 0.0 \mathrm{e}$ & - & $1.1 \pm 0.1 \mathrm{ij}$ \\
\hline & Mean & $0.2 \pm 0.1^{\mathrm{N} . \mathrm{S}}$ & $0.9 \pm 0.2 \mathrm{~A}$ & $3.2 \pm 0.5 \mathrm{~B}$ & $2.2 \pm 0.6 \mathrm{~B}$ & $0.5 \pm 0.1 \mathrm{~B}$ & - & $1.4 \pm 0.1 \mathrm{C}$ \\
\hline \multirow[t]{7}{*}{ S. graminum } & $\mathrm{V} 1$ & $0.1 \pm 0.0$ & $0.5 \pm 0.0 \mathrm{~b}$ & $12.3 \pm 0.2 \mathrm{~d}$ & $3.4 \pm 0.1 \mathrm{de}$ & - & - & $4.0 \pm 0.1 \mathrm{~h}$ \\
\hline & $\mathrm{V} 2$ & $0.3 \pm 0.2$ & $0.4 \pm 0.0 \mathrm{~b}$ & $14.2 \pm 0.0 \mathrm{~b}$ & $2.8 \pm 0.3 \mathrm{de}$ & - & - & $4.4 \pm 0.1 \mathrm{gh}$ \\
\hline & V3 & $0.3 \pm 0.2$ & $0.2 \pm 0.0 \mathrm{~b}$ & $13.0 \pm 0.1 \mathrm{c}$ & $10.7 \pm 0.1 \mathrm{~b}$ & - & - & $6.1 \pm 0.0 \mathrm{f}$ \\
\hline & V4 & $0.1 \pm 0.0$ & $0.3 \pm 0.0 \mathrm{~b}$ & $13.8 \pm 0.1 \mathrm{~b}$ & $15.4 \pm 0.2 \mathrm{a}$ & - & - & $7.4 \pm 0.0 \mathrm{e}$ \\
\hline & V5 & $0.0 \pm 0.0$ & $0.1 \pm 0.0 \mathrm{~b}$ & $16.8 \pm 0.1 \mathrm{a}$ & $9.3 \pm 0.4 \mathrm{c}$ & - & - & $6.6 \pm 0.1 \mathrm{f}$ \\
\hline & V6 & $0.3 \pm 0.1$ & $0.2 \pm 0.1 \mathrm{~b}$ & $7.00 \pm 0.1 \mathrm{e}$ & $11.4 \pm 0.2 \mathrm{~b}$ & - & - & $4.7 \pm 0.0 \mathrm{gh}$ \\
\hline & Mean & $0.2 \pm 0.1^{\mathrm{N} . \mathrm{S}}$ & $0.3 \pm 0.1 \mathrm{~B}$ & $12.9 \pm 1.8 \mathrm{~A}$ & $8.8 \pm 2.8 \mathrm{~A}$ & - & - & $5.5 \pm 0.7 \mathrm{~B}$ \\
\hline \multirow[t]{7}{*}{ S. avenae } & V1 & - & - & - & - & $25.2 \pm 0.2 \mathrm{a}$ & $4.2 \pm 0.2 \mathrm{a}$ & $14.7 \pm 0.2 \mathrm{a}$ \\
\hline & $\mathrm{V} 2$ & - & - & - & - & $24.9 \pm 0.5 \mathrm{a}$ & $1.7 \pm 0.5 \mathrm{~b}$ & $13.3 \pm 0.1 \mathrm{~b}$ \\
\hline & V3 & - & - & - & - & $17.9 \pm 0.4 \mathrm{~b}$ & $0.3 \pm 0.1 \mathrm{c}$ & $9.1 \pm 0.3 \mathrm{c}$ \\
\hline & V4 & - & - & - & - & $16.3 \pm 0.2 \mathrm{c}$ & $0.2 \pm 0.1 \mathrm{c}$ & $8.2 \pm 0.1 \mathrm{~d}$ \\
\hline & V5 & - & - & - & - & $9.1 \pm 0.3 \mathrm{~d}$ & $0.9 \pm 0.1 \mathrm{bc}$ & $5.0 \pm 0.2 \mathrm{~g}$ \\
\hline & V6 & - & - & - & - & $9.0 \pm 0.2 \mathrm{~d}$ & $0.8 \pm 0.0 \mathrm{bc}$ & $4.9 \pm 0.1 \mathrm{~g}$ \\
\hline & Mean & - & - & - & - & $17.1 \pm 4.1 \mathrm{~A}$ & - & $9.0 \pm 2.3 \mathrm{~A}$ \\
\hline \multicolumn{9}{|c|}{ ANOVA } \\
\hline \multicolumn{2}{|l|}{ Species } & 0.91 & 105.71 & 110.12 & 6230.11 & 14973.6 & - & 9208.62 \\
\hline \multirow{2}{*}{\multicolumn{2}{|c|}{ F (P) Tukey HSD }} & $0.4411^{\mathrm{N} . \mathrm{S}}$ & $0.0093 *$ & $0.000 *$ & $0.0000^{*}$ & 0.0000 & & $0.000 *$ \\
\hline & & 1.492 & 0.2550 & 0.1252 & 0.1810 & 0.295 & & 0.143 \\
\hline \multirow{3}{*}{\multicolumn{2}{|c|}{ F (P) Tukey HSD }} & 4.05 & 4.94 & 1053.39 & 339.19 & 410.56 & 31.46 & 218.77 \\
\hline & & $0.0106^{*}$ & $0.0042 *$ & $0.000 *$ & $0.0000 *$ & $0.0000^{*}$ & $0.0000^{*}$ & $0.000 *$ \\
\hline & & 0.3276 & 0.4354 & 0.2791 & 0.8087 & 0.8962 & 1.302 & 0.3924 \\
\hline \multicolumn{2}{|c|}{ Species $\times$ Var. } & 0.00 & 3.35 & 379.22 & 315.94 & 442.24 & - & 730.07 \\
\hline \multirow{2}{*}{\multicolumn{2}{|c|}{ F (P) Tukey HSD }} & $1.0000^{\mathrm{N} . \mathrm{S}}$ & $0.0232 *$ & $0.0000 *$ & $0.0000 *$ & $0.0000^{*}$ & & $0.000^{*}$ \\
\hline & & 0.4944 & 0.8178 & 0.4828 & 1.1217 & 1.4138 & & 0.6743 \\
\hline
\end{tabular}

V1, Galaxy-13; V2, Millat-11; V3, NW-1-8183-8; V4, NW3-3341-7; V5, Lasani-08; V6, Faisalabad -08; N.S, Non significant; * , significant at 0.05 $\alpha$; GS, growth stages of wheat plant; S, Seeding; 11, first leaf; 13, Three leaves; 21, Start of tillering stage; 25, five tillers; 29, End of tillering stage; 30, Start of stem elongation; 31, First node visible; 32, Second node visible; 37, Flag leaf just visible; 39, Flag leaf fully emerged; 49, Booting; 55, Ear 50\% emerged; 59, Ears fully emerged; 69, End of flowering; 89, Fully ripe. 


\section{Statistical analysis}

The data were analyzed following split plot design (Steel et al., 1997) with statistical software Statistix 8.1 (Analytical software, Statistix; Tallahassee, Florida, USA, 1985-2005). A repeated-measures ANOVA was also done considering number of observations as additional factor. Means were compared with Tukey HSD test of significance $(\mathrm{P}=0.05)$.

\section{RESULTS}

The incidence of aphids in negligibly low numbers was started on $19^{\text {th }}$ January when crop was at stem elongation stage (GS 30) on all wheat varieties/lines (Table I). The aphid population increased gradually with the growth of plants during reproductive stages. Peak infestations was earliest on $16^{\text {th }}$ February (GS 37: flag leaf just visible) on Lasani-08, on $23^{\text {rd }}$ February (GS 39: flag leaf fully emerged) on both NW-3-3341-7 and Faisalabad-08. On Galaxy-13, Millat-08, and NW-1-8183-8, peaks of aphids were noted on $2^{\text {nd }}$ March (GS 49: booting stage). Aphids started to decline on $9^{\text {th }}$ March (GS 55: 50\% emergence of ear).

From incidence (GS30: start of stem elongation) to decline (GS55: ear emergence stage), aphid complex comprised of three species (Table II) which were identified as Rhopalosiphum padi (L.), Schizaphis graminum (R.), and Sitobion avenae (F.). R. padi was dominant over population of $S$. graminum at GS 32 . The population of $S$. graminum increased gradually and peaked at flag leaf stage (GS 37) and became dominant over $R$. padi. The population of both $S$. graminum and $R$. padi started to decline at full emergence of flag leaf (GS 39). Third species, S. avenae appeared late at booting stage (GS 49). The population of $S$. avenae was higher than $R$. padi at GS 49. The population of $S$. avenae declined at GS 55 (with $50 \%$ ears emergence). During the entire season, mean population of three species i.e., R. padi, S. graminum, and S. avenae were 1.4, 5.5, and 9.0 aphids/tiller, respectively.

At GS 32 (with the visibility of $2^{\text {nd }}$ node) on $2^{\text {nd }}$ Feb., aphid population was observed non-significant for all the varieties and treatments (Table III). Maximum aphid number was observed on Millat-11 and minimum on Lasnai-08. On $9^{\text {th }}$ Feb., aphid infestation was found significant for varieties and treatments. The highest mean aphid population was observed on NW-3-3341-7 and the lowest mean aphid population was found on Galaxy-13 (1.0). Aphids were high in numbers at booting stage (GS 49).

First application of imidacloprid was done on $9^{\text {th }} \mathrm{Feb}$. (at GS32: visibility of $2^{\text {nd }}$ node) on all wheat varieties after aphid data collection (Table III). Aphid population was higher on Lasani-08 and lower in Faisalabad-08. Aphid population was lower under imidacloprid treated plots of all wheat cultivars while the highest under untreated regime.

Second application of imidacloprid was done on $23^{\text {rd }}$ Feb. (at GS39: full emergence of flag leaf) on all wheat varieties after aphid data collection (Table III). Aphid population at booting stage (GS 49) was significant on varieties and treatments. The highest aphid infestation was observed on Millat-11, while, the lowest was on Lasani-08 and Faisalabad-08.

Aphid infestation was the highest on untreated plots of Galaxy (26.0 aphids/tiller) and Millat-08 (25.7 aphids/ tiller). Aphid population was the lowest in NW-3-3341-7, Lasani-08 and Faisalabad-08 in imidacloprid treated plots. The overall mean seasonal infestation was higher on wheat line NW-3-3341-7 (6.4 aphid/tiller) and on NW-1-81838 (6 aphid/tiller). The lowest aphid population (4 aphid/ tiller) was observed on Faisalabad-08.

All wheat varieties/lines showed chlorophyll measurement value above 35 indicating that the crop was in good health (Table IV). Chlorophyll content and photosynthetic rate were statistically the highest in imidacloprid treated plots as compared to untreated plots.

Grain yield was observed statistically significant for all varieties and treatments. Maximum mean grain yield was observed in Galaxy which was at par with other wheat varieties Millat-11 and NW-1-8183-8. Lowest grain yield was observed in Lasani-08. The highest grain yield was found in imidacloprid treated plots of Galaxy-13 and the lowest in untreated plots of Lasani-08 (Table IV). The imidacloprid sprayed plots gave the highest yield as compared to untreated plot of all varieties. Similarly, percent yield loss was higher in Lasani-08 and lesser in Galaxy. Based on the mean seasonal aphid infestation and mean yield gain from all wheat varieties, Galaxy-13, Millat-11, NW-1-8183-8, NW-3-3341-7 and Faisalabad-08 were found tolerant against aphid damage. Wheat variety Lasani- 08 was found susceptible by having low yield even with low numbers of aphids.

Applications of imidacloprid reduced aphid infestation than untreated plots. The imidacloprid treated plots showed less aphid infestation, higher chlorophyll content and photosynthetic rate of all the tested varieties (Table IV). Due to heavy aphid infestations, plants of untreated plots showed low photosynthetic rate and chlorophyll content, resulting lower yield harvests than treated plots. Imidacloprid applications resulted in good return for better grain yield. Cost benefit ratios were higher in NW-1-8183-8 (1:9.2) and lower in NW-3-3341-7 and Lasani-08 (1:7.4). 
Table III.- Split plot analysis: Main plot (varieties) and subplots (treated and untreated plots): Mean aphid infestations (per tiller \pm S.E) during the crop season.

\begin{tabular}{|c|c|c|c|c|c|c|c|c|}
\hline Aphid & Varieties & $\begin{array}{c}2 \text { Feb } \\
\text { (GS32) }\end{array}$ & $\begin{array}{c}9 \text { Feb } \\
\text { (GS32) }\end{array}$ & $\begin{array}{l}16 \text { Feb } \\
\text { (GS37) }\end{array}$ & $\begin{array}{c}\text { 23Feb } \\
\text { (GS39) }\end{array}$ & $\begin{array}{c}\text { 2Mar } \\
\text { (GS 49) }\end{array}$ & $\begin{array}{c}\text { 9Mar } \\
\text { (GS55) }\end{array}$ & $\begin{array}{c}\text { Seasonal } \\
\text { Mean }\end{array}$ \\
\hline \multicolumn{9}{|c|}{ Main plot analysis } \\
\hline \multirow[t]{7}{*}{ Var. } & Galaxy-13 & $0.3 \pm 0.0$ & $1.0 \pm 0.4 \mathrm{c}$ & $7.8 \pm 0.2 \mathrm{c}$ & $3.5 \pm 0.3 \mathrm{~d}$ & $16.0 \pm 0.5 \mathrm{~b}$ & $4.0 \pm 0.2 \mathrm{a}$ & $5.4 \pm 0.2 \mathrm{c}$ \\
\hline & Millat-11 & $0.6 \pm 0.3$ & $1.7 \pm 0.1 \mathrm{abc}$ & $9.1 \pm 0.1 \mathrm{~b}$ & $3.6 \pm 0.4 \mathrm{~d}$ & $17.8 \pm 0.4 \mathrm{a}$ & $1.6 \pm 0.4 \mathrm{~b}$ & $5.7 \pm 0.1 \mathrm{~b}$ \\
\hline & NW-1-8183-8 & $0.4 \pm 0.2$ & $1.8 \pm 0.1 \mathrm{ab}$ & $8.2 \pm 0.1 \mathrm{c}$ & $12.1 \pm 0.2 \mathrm{~b}$ & $14.1 \pm 0.4 \mathrm{c}$ & $0.2 \pm 0.1 \mathrm{~b}$ & $6.1 \pm 0.1 \mathrm{a}$ \\
\hline & NW-3-3341-7 & $0.3 \pm 0.1$ & $2.0 \pm 0.1 \mathrm{a}$ & $8.8 \pm 0.1 \mathrm{~b}$ & $15.3 \pm 0.4 \mathrm{a}$ & $11.8 \pm 0.3 \mathrm{~d}$ & $0.2 \pm 0.1 \mathrm{~b}$ & $6.4 \pm 0.1 \mathrm{a}$ \\
\hline & Lasani-08 & $0.2 \pm 0.1$ & $1.2 \pm 0.5 \mathrm{abc}$ & $10.6 \pm 0.1 \mathrm{a}$ & $8.5 \pm 0.6 \mathrm{c}$ & $7.3 \pm 0.3 \mathrm{e}$ & $0.7 \pm 0.2 \mathrm{~b}$ & $4.8 \pm 0.1 \mathrm{~d}$ \\
\hline & Faisalabad-08 & $0.4 \pm 0.2$ & $1.1 \pm 0.4 \mathrm{bc}$ & $4.4 \pm 0.1 \mathrm{~d}$ & $9.3 \pm 0.3 \mathrm{c}$ & $7.6 \pm 0.2 \mathrm{e}$ & $1.1 \pm 0.5 \mathrm{~b}$ & $4.0 \pm 0.1 \mathrm{e}$ \\
\hline & Mean & $0.4 \pm 0.1 \mathrm{E}$ & $1.5 \pm 0.2 \mathrm{D}$ & $8.2 \pm 0.8 \mathrm{C}$ & $8.7 \pm 1.9 \mathrm{~B}$ & $12.5 \pm 1.8 \mathrm{~A}$ & $1.3 \pm 0.6 \mathrm{D}$ & - \\
\hline \multicolumn{9}{|c|}{ ANOVA } \\
\hline & Var. (F) & 0.78 & 6.25 & 454.41 & 243.83 & 314.60 & 21.65 & 255.37 \\
\hline & Var. (P) & $0.5835^{\text {N.S }}$ & $0.0070^{*}$ & $0.0000^{*}$ & $0.0000 *$ & $0.0000^{*}$ & $0.0000 *$ & $0.0000 *$ \\
\hline & Tukey HSD & 0.8011 & 0.8580 & 0.4749 & 1.4624 & 1.2105 & 1.5261 & 0.2755 \\
\hline & $\operatorname{Var} \times \mathrm{OBD}(\mathrm{F})$ & - & - & - & - & - & - & 200.89 \\
\hline & $\operatorname{Var} \times$ OBD $(\mathrm{P})$ & & & & & & & 0.0000 \\
\hline & Tukey HSD & & & & & & & - \\
\hline & $\operatorname{Var} \times \operatorname{Trt} \times$ OBD $(F)$ & - & - & - & - & - & - & 67.83 \\
\hline & Var $\times \operatorname{Trt} \times$ OBD $(\mathrm{P})$ & & & & & & & $0.0000^{*}$ \\
\hline & Tukey HSD & & & & & & & - \\
\hline \multicolumn{9}{|c|}{ Subplot analysis } \\
\hline \multirow[t]{6}{*}{$\mathrm{I}_{0}$} & Galaxy-13 & $0.2 \pm 0.1$ & $0.4 \pm 0.3 \mathrm{~b}$ & $15.3 \pm 0.3 \mathrm{~d}$ & $4.2 \pm 0.2 \mathrm{ef}$ & $26.0 \pm 0.4 \mathrm{a}$ & $4.2 \pm 0.2 \mathrm{a}$ & $8.4 \pm 0.2 \mathrm{~b}$ \\
\hline & Millat-11 & $0.7 \pm 0.3$ & $1.8 \pm 0.1 \mathrm{ab}$ & $17.8 \pm 0.1 \mathrm{~b}$ & $3.5 \pm 0.4 \mathrm{ef}$ & $25.7 \pm 0.5 \mathrm{a}$ & $1.7 \pm 0.5 \mathrm{~b}$ & $8.5 \pm 0.1 \mathrm{~b}$ \\
\hline & NW-1-8183-8 & $0.7 \pm 0.3$ & $1.2 \pm 0.1 \mathrm{ab}$ & $16.2 \pm 0.1 \mathrm{c}$ & $13.3 \pm 0.1 \mathrm{~b}$ & $18.5 \pm 0.5 \mathrm{~b}$ & $0.3 \pm 0.2 \mathrm{~b}$ & $8.4 \pm 0.1 \mathrm{~b}$ \\
\hline & NW-3-3341-7 & $0.2 \pm 0.1$ & $1.5 \pm 0.1 \mathrm{ab}$ & $17.3 \pm 0.1 \mathrm{~b}$ & $19.2 \pm 0.3 \mathrm{a}$ & $16.8 \pm 0.2 \mathrm{~b}$ & $0.2 \pm 0.1 \mathrm{~b}$ & $9.2 \pm 0.0 \mathrm{a}$ \\
\hline & Lasani-08 & $0.0 \pm 0.0$ & $0.7 \pm 0.4 \mathrm{~b}$ & $21.0 \pm 0.1 \mathrm{a}$ & $11.7 \pm 0.5 \mathrm{bc}$ & $9.3 \pm 0.3 \mathrm{c}$ & $0.9 \pm 0.1 \mathrm{~b}$ & $7.3 \pm 0.1 \mathrm{c}$ \\
\hline & Faisalabad-08 & $0.5 \pm 0.3$ & $0.8 \pm 0.4 \mathrm{ab}$ & $08.8 \pm 0.1 \mathrm{e}$ & $11.2 \pm 0.3 \mathrm{bc}$ & $9.3 \pm 0.2 \mathrm{c}$ & $1.1 \pm 0.5 \mathrm{~b}$ & $5.3 \pm 0.1 \mathrm{~d}$ \\
\hline \multirow[t]{6}{*}{$\mathrm{I}_{2}$} & Galaxy-13 & $0.3 \pm 0.1$ & $1.5 \pm 0.4 \mathrm{ab}$ & $0.2 \pm 0.1 \mathrm{f}$ & $2.8 \pm 0.3 \mathrm{f}$ & $6.3 \pm 0.5 \mathrm{~d}$ & $3.9 \pm 0.1 \mathrm{a}$ & $2.5 \pm 0.1 \mathrm{fg}$ \\
\hline & Millat-11 & $0.5 \pm 0.3$ & $1.7 \pm 0.1 \mathrm{ab}$ & $0.3 \pm 0.2 \mathrm{f}$ & $3.6 \pm 0.3 \mathrm{ef}$ & $9.9 \pm 0.3 \mathrm{c}$ & $1.6 \pm 0.3 \mathrm{~b}$ & $2.9 \pm 0.1 \mathrm{f}$ \\
\hline & NW-1-8183-8 & $0.2 \pm 0.1$ & $2.4 \pm 0.1 \mathrm{a}$ & $0.2 \pm 0.1 \mathrm{f}$ & $10.8 \pm 0.3 \mathrm{c}$ & $9.8 \pm 0.3 \mathrm{c}$ & $0.2 \pm 0.1 \mathrm{~b}$ & $3.9 \pm 0.1 \mathrm{e}$ \\
\hline & NW-3-3341-7 & $0.4 \pm 0.1$ & $2.5 \pm 0.1 \mathrm{a}$ & $0.4 \pm 0.2 \mathrm{f}$ & $11.2 \pm 0.5 \mathrm{bc}$ & $6.8 \pm 0.3 \mathrm{~d}$ & $0.2 \pm 0.1 \mathrm{~b}$ & $3.6 \pm 0.1 \mathrm{e}$ \\
\hline & Lasani-08 & $0.4 \pm 0.2$ & $1.7 \pm 0.5 \mathrm{ab}$ & $0.2 \pm 0.1 \mathrm{f}$ & $5.4 \pm 0.6 \mathrm{de}$ & $5.2 \pm 0.2 \mathrm{~d}$ & $0.6 \pm 0.3 \mathrm{~b}$ & $2.2 \pm 0.1 \mathrm{~g}$ \\
\hline & Faisalabad-08 & $0.4 \pm 0.1$ & $1.4 \pm 0.4 \mathrm{ab}$ & $0.1 \pm 0.1 \mathrm{f}$ & $7.4 \pm 0.3 \mathrm{~d}$ & $5.8 \pm 0.1 \mathrm{~d}$ & $1.0 \pm 0.5 \mathrm{~b}$ & $2.7 \pm 0.1 \mathrm{fg}$ \\
\hline \multirow[t]{2}{*}{ Trt. } & $\mathrm{I}_{0}$ & $0.4 \pm 0.2$ & $1.1 \pm 0.2$ & $16.1 \pm 1.6 \mathrm{~A}$ & $10.5 \pm 2.4 \mathrm{~A}$ & $17.6 \pm 3.0 \mathrm{~A}$ & $1.4 \pm 0.3$ & $7.8 \pm 0.1 \mathrm{~A}$ \\
\hline & $\mathrm{I}_{2}$ & $0.4 \pm 0.2$ & $1.9 \pm 0.2$ & $0.2 \pm 0.2 \mathrm{~B}$ & $6.9 \pm 1.4 \mathrm{~B}$ & $7.3 \pm 0.3 \mathrm{~B}$ & $1.2 \pm 0.2$ & $2.9 \pm 0.1 \mathrm{~B}$ \\
\hline \multicolumn{9}{|c|}{ ANOVA } \\
\hline & Trt. F & 0.08 & 16.09 & 51444.3 & 371.01 & 2847.11 & 1.15 & 4041.67 \\
\hline & Trt. P & $0.7887^{\text {N.S }}$ & $0.0017 *$ & $0.0000^{*}$ & $0.0000^{*}$ & $0.0000^{*}$ & $0.3053^{\text {N.S }}$ & $0.0000^{*}$ \\
\hline & Tukey HSD & 0.2210 & 0.4348 & 0.1522 & 0.4138 & 0.4206 & 0.2488 & 0.1665 \\
\hline & Var. $\times$ Trt. $F$ & 1.49 & 0.94 & 553.42 & 40.94 & 184.48 & 0.42 & 41.11 \\
\hline & Var $\times$ Trt P & $0.2647^{\mathrm{N} . \mathrm{S}}$ & $0.4896^{\mathrm{N} . \mathrm{S}}$ & $0.0000^{*}$ & $0.0000^{*}$ & $0.0000^{*}$ & $0.8229^{\mathrm{N} . \mathrm{S}}$ & $0.0000^{*}$ \\
\hline & Tukey HSD & 1.1803 & 1.7090 & 0.7412 & 2.1740 & 1.9573 & 1.9773 & 0.6189 \\
\hline
\end{tabular}

I , Untreated control; $I_{2}$, two imidacloprid sprays on $9^{\text {th }}$ Feb and $23^{\text {rd }}$ Feb; N.S, Non significant; * significant at $0.05 \alpha$; GS, growth stages of wheat plant; S, Seeding; 11, first leaf; 13, Three leaves; 21, Start of tillering stage; 25, five tillers; 29, End of tillering stage; 30, Start of stem elongation; 31, First node visible; 32, Second node visible; 37, Flag leaf just visible; 39, Flag leaf fully emerged; 49, Booting; 55, Ear 50\% emerged; 59; Ears fully emerged; 69 , End of flowering; 89, Fully ripe. 
Table IV.- A summary table on wheat aphid (per tiller), chlorophyll content, photosynthetic rate, grain yield and cost and benefit ratio.

\begin{tabular}{|c|c|c|c|c|c|c|c|}
\hline Aphid & Varieties & $\begin{array}{c}\text { Mean aphid } \\
\text { infestation/tiller }\end{array}$ & $\begin{array}{l}\text { Photosynthetic } \\
\text { rate }\end{array}$ & $\begin{array}{c}\text { Chlorophyll } \\
\text { contents }\end{array}$ & $\begin{array}{c}\text { Grain yield } \\
\text { (grams) }\end{array}$ & $\begin{array}{l}\text { Yield/ha. } \\
(40 \mathrm{Kg} / \mathrm{ha})\end{array}$ & $\begin{array}{c}\text { Cost: } \\
\text { Benefit }\end{array}$ \\
\hline \multirow[t]{6}{*}{$\mathrm{I}_{0}$} & Galaxy-13 & $8.4 \pm 0.2 \mathrm{~b}$ & $0.4 \pm 0.2$ & $56.0 \pm 0.1 \mathrm{~d}$ & $21.0 \pm 1.1 \mathrm{bcde}$ & 94.3 & - \\
\hline & Millat-11 & $8.5 \pm 0.1 \mathrm{~b}$ & $0.4 \pm 0.1$ & $55.3 \pm 0.1 \mathrm{de}$ & $19.4 \pm 0.7$ cdef & 87.1 & - \\
\hline & NW-1-8183-8 & $8.4 \pm 0.1 \mathrm{~b}$ & $0.7 \pm 0.1$ & $55.9 \pm 0.3 \mathrm{de}$ & $18.1 \pm 0.9$ defg & 81.3 & - \\
\hline & NW-3-3341-7 & $9.2 \pm 0.1 \mathrm{a}$ & $0.5 \pm 0.3$ & $56.3 \pm 0.3 \mathrm{~cd}$ & $15.5 \pm 0.6 \mathrm{fg}$ & 69.5 & - \\
\hline & Lasani-08 & $7.3 \pm 0.1 \mathrm{c}$ & $0.6 \pm 0.1$ & $52.5 \pm 0.5 \mathrm{f}$ & $13.0 \pm 0.3 \mathrm{~g}$ & 58.1 & - \\
\hline & Faisalabad-08 & $5.3 \pm 0.1 \mathrm{~d}$ & $0.5 \pm 0.1$ & $57.8 \pm 0.3 \mathrm{bc}$ & $17.4 \pm 1.1 \mathrm{efg}$ & 78.2 & - \\
\hline \multirow{6}{*}{$\mathrm{I}_{2}$} & Galaxy-13 & $2.5 \pm 0.1 \mathrm{fg}$ & $1.0 \pm 0.2$ & $61.9 \pm 0.2 \mathrm{a}$ & $26.3 \pm 1.5 \mathrm{a}$ & 119.5 & $1: 8.6$ \\
\hline & Millat-11 & $2.9 \pm 0.1 \mathrm{f}$ & $0.7 \pm 0.1$ & $61.3 \pm 0.2 \mathrm{a}$ & $25.0 \pm 0.6 \mathrm{ab}$ & 112.2 & $1: 8.5$ \\
\hline & NW-1-8183-8 & $3.9 \pm 0.1 \mathrm{e}$ & $0.7 \pm 0.3$ & $56.6 \pm 0.5 \mathrm{~cd}$ & $24.2 \pm 1.8 \mathrm{abc}$ & 108.3 & $1: 9.2$ \\
\hline & NW-3-3341-7 & $3.6 \pm 0.1 \mathrm{e}$ & $0.7 \pm 0.3$ & $58.5 \pm 0.3 \mathrm{~b}$ & $20.4 \pm 0.2$ bcdef & 91.4 & $1: 7.4$ \\
\hline & Lasani-08 & $2.2 \pm 0.1 \mathrm{~g}$ & $1.4 \pm 0.3$ & $54.4 \pm 0.3 \mathrm{e}$ & $17.8 \pm 0.2 \mathrm{defg}$ & 79.9 & $1: 7.4$ \\
\hline & Faisalabad-08 & $2.7 \pm 0.1 \mathrm{fg}$ & $0.5 \pm 0.1$ & $60.4 \pm 0.4 \mathrm{a}$ & $22.7 \pm 0.3 \mathrm{abcd}$ & 101.6 & $1: 8.0$ \\
\hline \multirow[t]{2}{*}{ Trt. } & $\mathrm{I}_{0}$ & $7.8 \pm 0.1 \mathrm{~A}$ & $0.5 \pm 0.2 \mathrm{~B}$ & $55.6 \pm 0.3 \mathrm{~B}$ & $17.4 \pm 1.2 \mathrm{~B}$ & - & - \\
\hline & $\mathrm{I}_{2}$ & $2.9 \pm 0.1 \mathrm{~B}$ & $0.8 \pm 0.2 \mathrm{~A}$ & $58.9 \pm 0.3 \mathrm{~A}$ & $22.8 \pm 1.3 \mathrm{~A}$ & - & - \\
\hline \multicolumn{8}{|c|}{ ANOVA } \\
\hline & Trt. F & 4041.67 & 8.50 & 356.45 & 101.57 & - & - \\
\hline & Trt. P & $0.0000 *$ & $0.0130 *$ & $0.0000^{*}$ & $0.0000^{*}$ & & \\
\hline & Tukey HSD & 0.1665 & 0.2327 & 0.3727 & 1.1621 & & \\
\hline & Var. $\times$ Trt. F & 41.11 & 1.36 & 27.74 & 0.13 & - & - \\
\hline & Var. $\times$ Trt P & $0.0000^{*}$ & $0.3043^{\text {N.S }}$ & $0.0000^{*}$ & $0.9832^{\text {N.S }}$ & & \\
\hline & Tukey HSD & 0.6189 & 0.9846 & 1.5773 & 5.2381 & & \\
\hline
\end{tabular}

$\mathrm{I}_{0}$, Untreated control; $\mathrm{I}_{2}$, two imidacloprid sprays on $9^{\text {th }}$ Feb and $23^{\text {rd }}$ Feb; N.S, Non significant; * , significant at $0.05 \alpha$.

\section{DISCUSSION}

Results of our studies are in line with those of the previous workers who reported aphid incidence on wheat crop during early crop stages (GS30: stem elongation) during month of January (Ali et al., 2015; Shafiq et al., 2015). The results are also similar to (Ahmed et al., 2015) who observed aphid incidence 55 days after sowing. Thereafter, the population of aphid increase at exponential rate and reached its peak on $16^{\text {th }}$ Feb., at GS37 (Flag leaf just visible) on Lasani-08. The peak population of aphids was recorded in February (Akhtar and Perveen, 2002; Ali et al., 2012, 2015). On wheat cultivars Galaxy-13, Millat-11 and NW-1-8183-8 the infestation of aphids was higher on $16^{\text {th }}$ Feb., to $2^{\text {nd }}$ Mar., at booting stage. Results are consistent to researchers, who have found peak infestation of aphid during late February (Ali et al., 2012; Tabasum et al., 2012). Occurrence of aphid peaks were also reported during March (Shahzad et al., 2013; Ahmed et al., 2015).

We observed less aphid's infestation in earlier growth stages of wheat crop. The results are similar to those researchers who found slower multiplication of aphid population at vegetative stages and gradual increase in population with the growth of plants (Riazuddin and Khattak, 2004; Ahmed et al., 2015; John et al., 2017). Both quality and quantity of plant produce are affected by crop growth stage (Ahmed and Nasir, 2001; Ahmed et al., 2015) which directly effects insect growth and development and insect fecundity (Yazdani and Agarwal, 1997).

Aphid populations started to decline at ear emergence stage on $9^{\text {th }}$ to $16^{\text {th }}$ Mar. Our findings are in consistent with Ahmed et al. (2015) and Ali et al. (2015) who reported that aphid population decline when the crop reached to maturity and population disappears in the last week of March. However, the results are quite contradictory that aphid incidence in February (Muhammad et al., 2013; Ahmed et al., 2015), peaked in April (Helmi and Rashwan, 2013) and decline in April (Shahid et al., 2012; Ahmed et al., 2015).

The results of the study are in agreed with various investigators, who identified that three aphid species i.e., R. padi, S. avenae and S. graminum, as major wheat insect-pests on different wheat varieties in Pakistan (Zeb et al., 2011; Shahid et al., 2012; Ali et al., 2015). Some studies showed only $S$. graminum and $R$. padi infestation on wheat cultivar (Akhtar and Perveen, 2002; Shahzad et al., 2013). While, other studies showed $R$. maidis, $R$. padi and $S$. avenae infest different wheat cultivars (Helmi and Rashwan, 2013). The results can also be compared with Ali et al. (2015), who found that $R$. padi dominates during vegetative growth stage and $S$. avenae dominates during reproductive crop stages. Results are also in line to the previous researchers who reported that $S$. graminum and 
R. padi showed maximum density in February which at the end shifted to the ears (Akhtar and Perveen, 2002).

The outcomes of our result are in conformity with those (Khattak et al., 2007; Ali et al., 2011) who found that resistance levels were varied in different wheat cultivars. Our results are inconsistent to those who reported serious aphids attack on Faisalabad-08 (Ahmed et al., 2015), reasons might be due to different locations of the experiment. They reported that mean seasonal aphids infestation/tiller was 34.55 aphids/tiller on Faisalabad-08 and 24.68 on Lasani-08. It was also reported that aphid population on Lasani-08 remained less than Faisalabad-08. While, our results showed less aphid infestation on Faisalabad-08 than Lasani-08. The results are in close conformity with those who reported that the variety Faisalabad-08 suffered the minimum grain weight loss and was found resistant as compared to other tested varieties (Wains et al., 2010; Ali et al., 2011). They also found that grain weight was lower on Lasani-08 under both treated and untreated condition. The results can be compared with Shafiq et al. (2015), who reported that Galaxy-2013 and Millat-2011 for having better varietal resistance against Schizaphis graminum. The results are similar to the findings of (Shafiq et al., 2015; Shafique et al., 2016), where Galaxy 2013 as resistant variety with minimum aphids per plant.

The present findings can be compared with those (Ahmed et al., 2001; Ali et al., 2011), who reported that the application of imidacloprid efficiently controls aphids. Treatment of Confidor 25WP@ @ 500 ml/ha controlled aphids infestation/tiller significantly and showed reduction in aphid density/tiller nearly to zero (Wains et al., 2010). The results can be compared with Migui and Lamb (2003), who reported all species of aphids (R. padi, S. avenae and $S$. graminum) survived and reproduced on all wheat varieties, and caused reduction in spike biomass compared to treated plots. The results are also comparable to Shafiq et al. (2015), who found that imidacloprid as the most effective and persistent insecticide with maximum aphid mortality at 14 days of post treatment.

Both the chlorophyll content and photosynthetic rate were the highest in imidacloprid treated plots and the lowest in untreated control. The results are in conformity with Razaq et al. (2014a), who reported that photosynthetic rate and chlorophyll was lower in aphid infested plants as compared to that in aphid protected plants of canola crop. Similar results were reported by Hussain et al. (2014) as aphid feeding on Brassica campestris crop caused abatement of photosynthetic rate. Photosynthetic rate of wheat are similar to researchers, who reported a reduction in the photosynthesis rate of the cotton plants when infested by whitefly (Lin et al., 1999). Photosynthetic rate of rice crop showed reduction in the photosynthesis rate of plants when fed by plant hopper (Watanabe and Kitagawa, 2000). These findings suggested that reduction in photosynthetic rate was related with some change in metabolism due to aphid infestation as observed recently in okra (Razaq et al., 2014b).

High yielding wheat cultivars were observed to be the most susceptible varieties to aphid infestations. We found similar trend in variety Galaxy-13 which was heavily infested by aphids but it gave the highest yield. The results are also at par (Shafique et al., 2016), who has sorted Galaxy-13 as moderately resistant wheat cultivar against wheat aphids with the highest grain yield as compared to other cultivars. The grain yield in present study is not comparable with those researchers who found that grain weight was the lowest in Faisalabad-08 and higher in case of Lasani-08 (Ahmed et al., 2015). It was reported that varieties attacked by aphid showed poorer grain weight and thus, yield elements and aphid infestation level has inverse relationship. Finding of Ali et al. (2011) matches to our results in case of Faisalabad-08, which had less aphid infestations and showed good yield.

Results can also be compared with Shahzad et al. (2013) where single as well as two imidacloprid sprays could significantly increase grain weight. In our results, two imidacloprid applications resulted in maximum costbenefit ratio in NW-1-8183-8 (1:9.2) and minimum in Lasani-08 (1:7.4). Findings of Aziz et al. (2013) supports that the application of imidacloprid resulted maximum cost-benefit ratio of 1:1.34 followed by neem seed kernel extract 5\% giving ratio as $1: 1.31$. Net economic benefits from aphid control (CRDC, 2010) averaged \$33-\$37/ha from an active seed treatment (imidacloprid) and welltimed spray of foliar insecticide (pirimicarb or dimethoate).

\section{CONCLUSION}

Applications of imidacloprid significantly control the aphid infestations on wheat crop. Photosynthetic rate and chlorophyll are significantly higher in treated plots. With higher grain yield, wheat varieties, Galaxy-13 Millat-11, NW-1-8183-8, NW-3-3341-7 and Faisalabad-08 are tolerant against aphid infestations. Wheat variety Lasani-08 was susceptible to aphid infestations and results in less grain yield.

\section{Statement of conflict of interest}

Authors have declared no conflict of interest.

\section{REFERENCES}

Aheer, G.M., Munir, M. and Ali, A., 2006. Screening of wheat cultivars against aphids in ecological 
conditions of district Mandi Bahaudin. J. agric. Res., 44: 55-58.

Ahmed, F. and Nasir, S., 2001. Varietal resistance of wheat germplasm against wheat aphid (Sitobion avenae F.). Pak. Entomol., 23: 5-7.

Ahmed, N., Kanan, H., Inanaga, S., Ma, Y. and Sugimoto, Y., 2001. Impact of pesticide seed treatments on aphid control and yield of wheat in the Sudan. Crop Protec., 20: 929-934. https://doi. org/10.1016/S0261-2194(01)00047-3

Ahmed, T., Aslam, M., Hussan, M.W. and Iqbal, J., 2015. Aphids (Schizaphis graminum R.) infestation on different wheat (Triticum aestivum L.) varieties and their comparative yields. J. agric. Res., 53: 209-216.

Akhtar, L.H., Hussain, M., Iqbal, R.M., Amer, M. and Tariq, A.H., 2010. Losses in grain yield caused by Russian wheat aphid Diuraphis noxia (Mordvilko). Sarhad J. Agric., 26: 625-628.

Akhtar, M.S. and Perveen, S., 2002. Studies on population of wheat aphids on wheat crop in new campus area, Lahore. Punjab Univ. J. Zool., 17: 1422.

Akhtar, N., Ehsan-ul-Haq and Masood, M.A., 2006. Categories of resistance in national uniform wheat yield trials against Schizaphis graminum (Rondani) (Homoptera: Aphididae). Pakistan J. Zool., 38: 167-171.

Ali, A., Malik, H., Islam, N., Rafique, S., Saleem, M. and Hassan, M., 2012. Varietal variation and interaction between population of aphids and their predators in wheat. J. agric. Res., 50: 209-216.

Ali, I., Khan, B.S., Sagher, M. and Ali, A., 2011. Determination of varietal resistance for and losses by aphids in wheat cultivars. Pak. Entomol., 33: 157-160.

Ali, S., Khalid, S., Ali, S., Akhtar, K., Khan, M.S., Ali, A., Samad, A., Hussain, S., Fahad, S. and Khan, F., 2015. Preferential influence of wheat genotypes on the distribution pattern and population dynamics of cereal aphids and their natural enemies in Peshawar valley. Pakistan J. Zool., 47: 223-233.

Anonymous, 2015. Pakistan economic survey 2014-15. Available at: http:/www.finance.gov.pk/survey/ chapters_15/Highlights.pdf (Nov.15 2015).

Ashraf, M.Y., Khan, A.H. and Azmi, A.R., 1992. Cell membrane stability and its relation with some physiological processes in wheat. Acta Agron. Hung., 41: 183-183.

Aziz, M., Ahmad, M., Nasir, M. and Naeem, M., 2013. Efficacy of different neem (Azadirachta indica) products in comparison with imidacloprid against english grain aphid (Sitobion avenae) on wheat. Int. J. Agric. Biol., 15: 279-284.

Blackman, R. and Eastop, V., 2000. Aphids on the World's crops: An identification and information guide, $2^{\text {nd }}$ Ed. Wiley \& Sons, England.

Brewer, M.J. and Elliott, N.C., 2004. Biological control of cereal aphids in North America and mediating effects of host plant and habitat manipulations. Annu. Rev. Ent., 49: 219-242. https://doi. org/10.1146/annurev.ento.49.061802.123149

Burd, J.D. and Elliott, N.C., 1996. Changes in chlorophyll a fluorescence induction kinetics in cereals infested with Russian wheat aphid (Homoptera: Aphididae). J. econ. Ent., 89: 1332-1337.

Ciepiela, A.P., 1993. Harmful effect of the cereal aphid on winter wheat crops. Ochrona Roslin, 37: 9-10.

CRDC, 2010. Cereal aphids factsheet. Grains Research and Development Corporation, pp. 1-4.

FAOSTAT, 2015. Food and agricultural commodities production/Countries by commodity. Available at: http://faostat3.fao.org/browse/rankings/countries_ by_commodity/E (Nov.21, 2015).

Helmi, A. and Rashwan, R., 2013. Effect of wheat cultivars and sown dates on aphid infestation in Egypt. Mun. Ent. Zool., 8: 825-830.

Hussain, A., Razaq, M., Shahzad, W., Mahmood, K. and Khan, F.Z.A., 2014. Influence of aphid herbivory on photosynthetic parameters of Brassica compestris at Multan, Punjab, Pakistan. J. Biol. environ. Sci., 5: 410-416.

Iqbal, J., 2003. Role of physico-morphic plant characters towards resistance against aphids on wheat. $\mathrm{Ph}$. D. thesis, University of Agriculture, Faisalabad, Pakistan.

Jarosik, V., Honek, A. and Tichopad, A., 2003. Comparison of field population growths of three cereal aphid species on winter wheat. Pl. Prot. Sci., 39: 61-64.

John, F., Saeed, N.A., Nadeem, S. and Hamed, M., 2017. Integration of planting time and insecticide to manage aphid infestations in wheat for better crop productivity. Pakistan J. Zool., 49: 1343-1351. http://dx.doi.org/10.17582/journal. pjz/2017.49.4.1343.1351

Khan, A.A., Khan, A.M., Tahir, H.M., Afzal, M., Khaliq, A., Khan, S.Y. and Raza, I., 2011. Effect of wheat cultivars on aphids and their predator populations. Afri. J. Biotechnol., 10: 18399-18402.

Khan, A.M., Khan, A.A., Afzal, M. and Iqbal, M.S., 2012. Wheat crop yield losses caused by the aphids infestation. J. Biofertil. Biopestic., 3: 122.

Khan, M.R., Anjum, F.M., Zahoor, T. and Nawaz, 
H., 2009. Biochemical and Technological characterization of Pakistani spring wheats. Pak. J. agric. Sci., 46: 2076-2086.

Khan, S.A., 2005. Studies on the aphids distribution pattern and their natural enemies in wheat and maize crop. Ph. D. thesis, KP Agricultural University, Peshawar.

Khattak, M.K., Riazuddin and Anayatullah, M., 2007. Population dynamics of aphids (Aphididae: Homoptera) on different wheat cultivars and response of cultivars to aphids in respect of yield and yield related parameters. Pakistan J. Zool., 39: 109-115.

Laycock, D., 2004. Manual of field trials in crop protection, $4^{\text {th }}$ Ed. Syngenta International, AG Basel, Switzerland.

Lin, T.B., Schwartz, A. and Saranga, Y.E., 1999. Photosynthesis and productivity of cotton under silverleaf whitefly stress. Crop Sci., 39: 174-184. https://doi.org/10.2135/ cropsci1999.0011183X003900010028x

Liu, S.Y., Stoltz, R.L. and Niu, Z.Z., 1986. Damage to wheat by Macrosiphum avenae in Northwest China. J. econ. Ent., 79: 688-699.

Migui, S. and Lamb, R., 2003. Patterns of resistance to three cereal aphids among wheats in the genus Triticum (Poaceae). Bull. entomol. Res., 93: 323333. https://doi.org/10.1079/BER2003246

Muhammad, W., Nasir, M., Abbas, S.K. and Irshad, M., 2013. Resistance pattern against aphid (Diuraphis noxia) in different wheat varieties/lines at District Layyah. Acad. J. Ent., 6: 116-120.

Razaq, M., Abbas, G., Farooq, M. and Athar, H.R., 2014a. Effect of insecticidal application on aphid population, photosynthetic parameters and yield components of late sown varieties of Canola, Brassica napus L. Pakistan J. Zool., 46: 661-668.

Razaq, M., Haneef, Q., Athar, H.R., Nasir, M. and Afazal, M., 2014b. Interactive effect of nitrogen and on jassid (Amrasca devastans Dist.) population and photosynthetic capacity of okra Abelmoschus esculentus (L.) Moench. Pakistan J. Zool., 46: $577-$ 579.

Riazuddin, M.A. and Khattak, K., 2004. Screening resistant wheat lines against aphids. Pak. Entomol., 26: $13-18$.

Riedell, W.E., Kieckhefer, R.W., Langham, M.A. and Hesler, L.S., 2003. Root and shoot responses to bird cherry-oat aphids and in spring wheat. Crop Sci., 43: 1380-1386. https://doi.org/10.2135/ cropsci2003.1380

Shafiq, M.A., Khan, R.R., Shakeel, M., Akhter, M.J., Waqas, M.A., Anees, M.U. and Gull, U., 2015. Sieving varietal resistance in bread wheat cultivars and chemical control efficacy against Schizaphis graminum R. J. Adv. Bot. Zool., 3: 1-6.

Shafique, M.A., Ahmed, K.S., Haider, N., Khan, R.R. and Majeed, M.Z., 2016. Field evaluation of different insecticides against wheat aphid ( $S$. graminum R.) and comparative yield assessment for different wheat cultivars. Acad. J. Ent., 9: 1-7.

Shahid, S., Zia, A., Naeem, M. and Naz, F., 2012. Bionomics of aphids and their parasitoid in selected wheat varieties grown under homologous ecological conditions. Pakistan J. Zool., 44: 14231430.

Shahzad, M.W., Razaq, M., Hussain, A., Yaseen, M., Afzal, M. and Mehmood, M.K., 2013. Yield and yield components of wheat (Triticum aestivum L.) affected by aphid feeding and sowing time at Multan, Pakistan. Pakistan J. Bot., 45: 2005-2011.

Steel, R.G.D., Torrie, J.H. and Dickey, D.A., 1997. Principles and procedures of statistics. A biometrical approach, $3^{\text {rd }}$ ed. McGraw Hill Inc., New York.

Tabasum, S., Noorka, I.R., Afzal, M. and Ali, A., 2012. Screening best adopted wheat lines against aphid (Schizaphis graminum Rondani) population. Pak. Entomol., 34: 51-53.

Trdan, S. and Milevoj, L., 1999. The cereal aphid (Sitobion avenae F.) wheat pest. Sodobno Kmetijstvo, 32: 119-128.

Wains, M.S., Ali, M., Hussain, M., Anwar, J., Zulkiffal, M. and Sabir, W., 2010. Aphid dynamics in relation to meteorological factors and various management practices in bread wheat. J. Pl. Prot. Res., 50: 386392. https://doi.org/10.2478/v10045-010-0065-6

Watanabe, T. and Kitagawa, H., 2000. Photosynthesis and translocation of assimilates in rice plants following phloem feeding by the planthopper Nilaparvatalugens (Homoptera: Delphacidae). J. econ. Ent., 93: 1192-1197.

Yazdani, S.S. and Agarwal, M.L., 1997. Elements of insect ecology. Narosa Publishing House, New Dehli.

Zeb, Q., Badshah, H., Ali, H., Shah, R. and Rehman, M., 2011. Population of aphids on different varieties/lines of wheat and their effect on yield and thousands grain weight. Sarhad J. Agric., 27: $443-450$. 